\title{
A importância das políticas públicas para o tratamento da hemoglobinúria paroxística noturna
}

\author{
The importance of public policies for the treatment of paroxysmal nocturnal hemoglobinuria
}

\author{
Beatriz Boni de Mattos ${ }^{\bullet}$, Rodrigo Vinícius de Souza Carvalho* ${ }^{\bullet}$, Anderson Scherer ${ }^{\bullet}$ \\ Universidade Anhembi Morumbi, São Paulo, São Paulo, Brasil. *Autor para correspondência. E-mail: rviniciuscarvalho@uol.com.br
}

\begin{abstract}
Resumo: Introdução: Doença rara é classificada como aquela que afeta até 65 pessoas em cada 100.000 indivíduos. Normalmente são condições crônicas e degenerativas, muitas delas sem cura ou tratamento efetivo e acessível, que causa um alto grau de morbimortalidade, além de enfrentarem um grande desafio, pois devido a sua baixa incidência, os profissionais de saúde não possuem o conhecimento sobre a sua existência, o que reflete em um diagnóstico tardio e falta de preparo da saúde pública. Revisão: Esse artigo, possui o foco na doença rara denominada hemoglobinúria paroxística noturna (HPN) e o seu cenário na saúde pública. Discussão: A HPN é caracterizada como uma anemia hemolítica crônica adquirida rara, causada pela expansão clonal não-maligna de uma ou várias células-tronco hematopoiéticas, desenvolvendo variados sintomas em seus portadores. Sua incidência anual estimada é de 1,3 novos casos por um milhão de indivíduos, mas por ser uma doença de difícil diagnóstico, o número pode estar subestimado e não refletir a realidade. Considerações finais: Os pacientes de HPN enfrentam uma longa e difícil trajetória, desde o seu diagnóstico até o efetivo tratamento. Mesmo com diretrizes estabelecidas, muito pacientes portadores de doenças raras e seus respectivos familiares, recebem tratamento desigual no serviço de saúde. Políticas públicas são necessárias e precisam ser efetivas para melhorar o acesso à informação e tratamento, fornecer estrutura e acompanhamento necessário, para dessa forma, reduzir o impacto causado por essas doenças e contribuir para a melhoria da qualidade de vida dos pacientes.
\end{abstract}

Palavras-chave: doença rara, hemoglobinúria paroxística noturna, HPN, saúde pública, sistema único de saúde, SUS.

\begin{abstract}
Introduction: Rare disease is classified as one that affects up to 65 people per 100,000 individuals. They are usually chronic and degenerative conditions, many of them without a cure or effective and accessible treatment, which cause a high degree of morbidity and mortality, in addition to facing a great challenge, because due to their low incidence, health professionals do not have the knowledge about their existence, which reflects in a late diagnosis and lack of public health preparation. Review: This article focuses on the rare disease called Paroxysmal Nocturnal Hemoglobinuria (PNH) and its scenario in public health. Discussion: The PNH is characterized as a rare acquired chronic hemolytic anemia, caused by the non-malignant clonal expansion of one or several hematopoietic stem cells, developing various symptoms in their carriers. Its estimated annual incidence is 1.3 new cases per one million individuals, but as it is a difficult disease to diagnose, the number may be underestimated and does not reflect reality. Final considerations: PNH patients face a long and difficult trajectory, from diagnosis to effective treatment. Even with established guidelines, many patients with rare diseases and their respective families receive unequal treatment in the health service. Public policies are necessary and need to be effective to improve access to information and treatment, provide the necessary structure and follow-up, to reduce the impact caused by these diseases and contribute to improving the quality of life of patients.
\end{abstract}

Keywords: rare disease, paroxysmal nocturnal hemoglobinuria, PNH, public health, unified health system, SUS.

\section{Introdução}

As doenças raras são definidas por aquela que afeta até 65 pessoas em cada 100.000 indivíduos (Ministério da Saúde, 2014). Normalmente são condições crônicas e degenerativas, muitas delas sem cura ou tratamento efetivo e acessível, elevando a morbimortalidade (Aureliano, 2018).

Com diagnóstico e tratamento complexos, muitas vezes, os pacientes não possuem acesso a um bom serviço de saúde, com profissionais de qualidade e eficazes, podendo gerar um alto impacto emocional na vida do paciente e de seus familiares (Anderson et al., 2013). 
Entre as doenças classificadas como rara, temos a hemoglobinúria paroxística noturna (HPN), que é uma anemia hemolítica crônica adquirida, que se origina das células tronco hematopoiéticas (CTHs), caracterizada por hemólise intravascular (Arruda et al., 2010).

Segundo a Portaria Conjunta $\mathrm{n}^{\mathrm{o}}$ 18, de 20 de novembro de 2019, recuperado em: http://conitec.gov.br/images/Relatorios/Portaria/2019/PortariaConjunta_SCTIE_SAES_18_2019.pd, incidência anual é estimada em 1,3 novos casos por um milhão de indivíduos. Como uma doença adquirida, ela pode ocorrer em qualquer idade, porém, a maioria dos pacientes recebem o diagnóstico entre a quarta e quinta década de vida (Arruda et al., 2010).

No cenário de uma doença rara, a falta de acesso a informações gera um alto impacto na vida dos pacientes e seus familiares, sendo uma experiência de inúmeros desafios, principalmente, na relação com os serviços de saúde, no qual será necessário por toda a vida (Luz et al., 2015).

Nesse artigo, será analisada a jornada do diagnóstico e tratamento dos pacientes de hemoglobinúria paroxística noturna. Serão levantados todos os pontos e desafios dessa trajetória, para que sejam evidenciadas a importância do acesso e efetividade da saúde pública na trajetória do paciente.

\section{Revisão}

Existem cerca de 7 mil doenças raras descritas, sendo aproximadamente $80 \%$ de origem genética e $20 \%$ de origem infecciosa, virais ou degenerativas (Ministério da saúde - Dia Mundial das Doenças Raras, https://bvsms.saude.gov.br/28-02-dia-mundial-das-doencas-raras). Segundo dado publicado pela Interfarma em 2018, no Brasil, estima-se a aproximadamente 13 milhões de pessoas acometidas por doenças raras.

Devido à falta de conhecimento, provavelmente pela baixa incidência, a maioria das doenças são diagnosticadas tardiamente, o que pode prejudicar o seu prognóstico e proporcionar consequências severas (Pfizer, “Doenças Raras - Quais são e por que são chamadas dessa forma?”, 2019).

As doenças raras podem ser vistas como um problema para a saúde pública, pois apesar de possuir uma baixa prevalência, as despesas para o diagnóstico e tratamento de um paciente portador de doença rara, gera um alto impacto para o orçamento público, principalmente em países que estão em desenvolvimento, que possuem recursos financeiros limitados (Pirett et al., 2017).

O Ministério da Saúde instituiu por meio da Portaria no 199 (Brasil, 2014) a "Política Nacional da Atenção Integral às Pessoas com Doenças Raras no Sistema Único e Saúde - SUS”, estabelecendo diretrizes para a atenção integral aos portares de doenças raras no âmbito da saúde pública brasileira, considerando incentivos financeiros para o custeio de medicamentos e tratamentos, melhorando o acesso aos serviços de saúde e à informação, contribuindo para a melhoria da qualidade de vida das pessoas com doenças raras. Segundo dado do Ministério da Saúde - Doenças raras: o que são, causas, tratamento, diagnóstico e prevenção (https://antigo.saude.gov.br/saude-de-a-z/doencas-raras, recuperado em agosto de 2021), o Brasil conta atualmente com 17 estabelecimentos habilitados e especializados para o atendimento de doenças raras, distribuídos em diversas unidades federativas do Brasil.

Mesmo com diretrizes estabelecidas, o Brasil enfrenta um grande e sério problema na disponibilidade de acesso ao tratamento para essas doenças, que em sua grande maioria, são tratadas com o os medicamentos denominados como "órfãos", que são aqueles destinados a doenças potencialmente fatais ou muito graves ou de perturbações raras, que devido à baixa incidência na população total, a rentabilidade dos medicamentos aos detentores é baixa, frente ao investimento adquirido para a pesquisa e o desenvolvimento do produto. De quase 50.000 medicamentos produzidos, apenas $10 \%$ são medicamentos órfãos, com vendas anuais de, aproximadamente, 100 bilhões de dólares (Pirett et al., 2017). Segundo a Lei N ${ }^{\circ} 10.938$, de 19 de outubro de 2001, os medicamentos órfãos são classificados como medicamentos especiais ou de alto custo. No Brasil, a grande maioria dos medicamentos de alto custo não são incorporados no SUS, o que dificulta ainda mais o acesso a medicação, fazendo com que o paciente necessite de judicialização para ter acesso ao tratamento adequado (Pirett et al., 2017).

Quantificar a prevalência da doença rara é difícil, devido as dificuldades do seu diagnóstico, mas mesmo que pouco prevalentes, considerando individualmente, acabam sendo mais frequentes, então devem ser tratadas como um problema de saúde pública.

\section{Hemoglobinúria paroxística noturna: HPN}

A hemoglobinúria paroxística noturna é considerada uma doença rara, com incidência anual estimada de 1,3 novos casos por um milhão de indivíduos e que causa um elevado índice de mortalidade. 
Fisiopatologicamente, a HPN é uma doença crônica grave, consequência da expansão clonal não-maligna de um ou várias células-tronco hematopoiéticas que adquiriram uma mutação somática no gene da fosfaditilinositolglicana classe-A (phosphatidyl inositol glycan-class A, PIG-A). O PIG-A é necessário para a síntese de âncoras de glicosil-fosfaditilinositol (GPI), que são estruturas essenciais para manter dezenas de proteínas, com diferentes funções, aderidas na membrana plasmática das células (Arruda et al., 2010; Brodsky, 2008).

Com a mutação no gene PIG-A, ocorre o bloqueio na síntese da âncora de GPI, causando a ausência da âncora na superfície celular, impedindo a ligação de diversas proteínas, entre elas, a CD55 (ou decay accelerating factor) e CD59 (ou membrane inhibitor of reactive lysis), que são inibidoras da cascata do complemento. Existem três caminhos por qual complemento é ativado: a via clássica, a via da lectina e a via alternativa, que resultam na geração de C3 complexos de convertase que mediam a clivagem de C3 a C3a e C3b (Mathieu et al.,1995). O CD55 inibe o complemento no nível de C3, já o CD59 é uma glicoproteína que interage diretamente com o complexo de ataque à membrana (também chamado de complexo de complemento terminal). A ausência de CD59 na superfície dos eritrócitos (hemácias), aderido a âncora e GPI, leva a formação descontrolada do ataque à membrana (MAC), o que os torna susceptíveis à quebra mediada pelo complexo do complemento terminal, o que explica a hemólise intravascular crônica, sendo a manifestação clínica primária na HPN. A deficiência dessas proteínas pode ser considerada como parcial, sendo células HNP tipo II, ou total, sendo células HPN tipo III com ausência total de proteínas (Arruda et al., 2010; Brodsky, 2008).

A maioria das manifestações clínicas da HPN são facilmente explicadas pela depleção tecidual do óxido nítrico. A hemoglobina livre no plasma tem grande afinidade pelo óxido nítrico e o remove da circulação, o que pode levar à distonia do músculo liso, ativação e agregação plaquetária (Ehlert et al., 2021). Células HPN são vulneráveis à ativação do complemento por qualquer das três vias, mas a via alternativa mantém um estado de ativação contínua, o que explica o porquê os pacientes com HPN têm hemólise crônica contínua (Brodsky, 2008).

A doença é classificada clinicamente, considerando sua apresentação, as manifestações clínicas, as características da medula óssea e o tamanho do clone mutante, podendo ser classificada como HPN clássica, HPN associada a síndromes de falência medular e HPN subclínica (Ehlert et al., 2021).

Em um estudo realizado com 856 pacientes que apresentaram manifestações da doença, foi constatado que os sintomas mais típicos da HPN são fadiga (80\%), dispneia (64\%), dor de cabeça (63\%), dor abdominal (44\%) e hemoglobinúria (62\%) (Schrezenmeier et al., 2014). Na análise epidemiológica de 385 pacientes com HPN dos EUA e Japão, 88\% apresentaram anemia (Nishimura et al., 2004). Além dos sintomas, estudos indicam que $67 \%$ das mortes de pacientes com HPN são devidas a eventos trombóticos e $18 \%$ são causadas por falência renal (Hillmen et al., 2013). O rápido reconhecimento dos sinais e sintomas da HPN e o diagnóstico precoce são vitais para o manejo da doença e manutenção da saúde do paciente (Jang et al., 2016; Borowitz et al., 2010).

Entre os métodos de diagnósticos, está a análise de citometria de fluxo (CF), que realiza a detecção de antígenos ancorados por GPI em células hematopoiéticas, sendo o ensaio informativo mais sensível para o diagnóstico e medição do tamanho do clone da HPN (Ehlert et al., 2021).

O eculizumabe é o principal fármaco indicado para o tratamento da HPN, considerado promissor pois retomou a qualidade de vida dos portadores da doença.

A substância é um anticorpo monoclonal humanizado, inibidor do complemento terminal que se liga de forma específica à proteína $\mathrm{C} 5$ do complemento, com alta afinidade, inibindo, deste modo, a sua clivagem em C5a e C5b e impedindo a geração do complexo de ataque à membrana (C5b-9) do complemento terminal. Inibindo o complemento terminal, o eculizumabe reduz rapidamente a hemólise intravascular, que é o principal fator de mortalidade. Reduz em até $92 \%$ os eventos trombóticos e, além disso, diminui significativamente as necessidades transfusionais em pacientes com HPN (SOLIRIS ${ }^{\circledR}$ (eculizumab) Bula do Profissional de Saúde (Bulário Eletrônico. Agência Nacional de Vigilância Sanitária (Anvisa), 2021, http://www.anvisa.gov.br/datavisa/fi la_bula/index.asp.).

Estudos apontam 92\% de sobrevida geral em 6 anos de pacientes com HPN em uso de eculizumabe, além disso, o eculizumabe permite ao paciente uma sobrevida global similar à da população geral pareada por idade e sexo (Hillmen et al., 2007).

No Brasil, o Soliris ${ }^{\circledR}$ (eculizumabe) foi registrado pela Anvisa em 13 de março de 2017. A grande desvantagem da medicação é o seu valor no mercado, que em março de 2018, foi publicado pela Anvisa que o preço máximo da venda do medicamento para o governo é de $\mathrm{R} \$ 11.942,60$, ultrapassando o valor de $\mathrm{R} \$ 800,00$ por paciente no ano. 


\section{Discussão}

Cerca de 6 a 10\% de todas as doenças do mundo são consideradas raras, estimando 7 mil doenças raras já identificadas (Pinto et al., 2019), incluindo a hemoglobinúria paroxística noturna. Os impactos econômicos, nas diversas famílias, frente ao tratamento e aos cuidados podem gerar grandes proporções negativas.

A importância das políticas públicas para as condições apresentadas neste artigo, impactam não somente na saúde do paciente, mas também a saúde das pessoas próximas ao portador. Em pesquisa realizada em 2019 com 99 famílias, considerando pacientes portadores de doenças raras atendidos em um hospital de referência no estado do Rio de Janeiro, contabilizou que, em sua totalidade, $54 \%$ das famílias não recebem nenhum benefício governamental, retirando do orçamento familiar para arcar com as necessidades do tratamento e da manutenção da saúde. Além disso, a situação gera apreensão, levando cerca de $69 \%$ dos entrevistados a solicitarem empréstimos bancários para financiar tratamentos e medicamentos (Pinto et al., 2019).

Garantido pela Constituição Federal de 1988, o Sistema Único de Saúde (SUS), é a expectativa de diagnóstico, tratamento e cuidado para diversas situações que acometem a saúde, incluindo os portadores de HPN, que em sua maioria, dependem do SUS para o tratamento.

Algumas dificuldades surgem durante o tratamento, devido a valores elevados das tecnologias de diagnósticos e dos medicamentos. De acordo com a Comissão de Direitos Humanos e Minorias da Câmara dos Deputados de Brasília, de setembro de 2017 a junho de 2018, a Associação de Famílias, Amigos e Portadores de Doenças Graves Raras (AFAG) apontou o óbito de 15 portadores de HPN, devido a complicações envolvendo a falta dos tratamentos oferecidos pelo governo federal, e consequentemente pelo SUS.

A elevada necessidade de saúde, insuficiência de tratamentos efetivos, políticas para acesso aos medicamentos e a adesão a consulta pública realizada, geraram a necessidade da elaboração de um protocolo para atender a demanda. O Ministério da Saúde, em 20 de novembro de 2019, instituiu a Portaria nº .18 , que aprovou e instaurou o Protocolo Clínico e Diretrizes Terapêuticas da hemoglobinúria paroxística noturna. Os pacientes portadores de HPN, a partir da data de publicação do protocolo, passaram a contar com o SUS para o transplante de células-tronco, exames para os diagnósticos da comorbidade e a vacina meningocócica ACWY que, segundo a bula do medicamento, é necessária para iniciar o tratamento (https://antigo.saude.gov.br/noticias/agencia-saude/46149-doenca-rara-que-afeta-o-sistema-sanguineoganha-tratamento-individualizado-no-sus).

Para os pacientes de HPN, a citometria de fluxo é considerada o padrão ouro para o diagnóstico laboratorial e, desde o dia 21 de novembro de 2019, foi publicada na Portaria $\mathrm{n}^{\circ}$. 61, a decisão de incorporar ao SUS o uso da citometria para o diagnóstico de HPN. Porém, o acesso ao exame pelo SUS é possível há apenas um ano e dez meses, anteriormente a esse período, os pacientes não tinham acesso ao exame gratuito, o que dificultava o seu diagnóstico, tornando-se tardio e diminuindo a qualidade de vida do paciente (Brasil, 2019).

O único tratamento curativo registrado e cientificamente comprovado para HPN é ofertado pelo SUS. O transplante de células-tronco hematopoéticas alogênico (TCTH-AL) é considerado a primeira opção para o tratamento de crianças e adolescentes acometidos, por possuírem uma melhor resposta ao tratamento, devido a idade. Para a elegibilidade ao transplante, o Protocolo Clínico considera elegíveis pacientes com fatores de risco para gradativa evolução da doença, podendo ocasionar a morte.

O eculizumabe é o principal fármaco indicado para o tratamento da HPN. O acesso ao medicamento via Sistema Público de Saúde se desenvolveu a partir da Portaria $\mathrm{n}^{\circ}$. 77, de 14 de dezembro de 2018, que incorporou no Sistema Único de Saúde o medicamento eculizumabe para o tratamento de paciente com hemoglobinúria paroxística noturna (Brasil, 2018). A incorporação, devido ao alto preço do medicamento no mercado, foi mediante condições, sendo a mais impactante, a negociação para a redução significante do preço do medicamento, além da reavaliação do paciente a cada 3 anos e o laudo próprio, emitido por profissional habilitado, para a dispensação do medicamento.

O crescente aparecimento de novas doenças raras é um importante problema de saúde pública, principalmente em países subdesenvolvidos, como o Brasil. A falta de incentivos governamentais para os fabricantes locais de medicamentos para tratamento de doenças raras afeta o desenvolvimento de estudos e tecnologias. No Artigo $8^{\circ}$ da Portaria ${ }^{\circ}$. 199, do Governo Federal, instaura a responsabilidade comuns dos órgãos públicos atribuídos a saúde a promoção ao intercâmbio de experiências e estímulo do desenvolvimento de estudos de pesquisa para o desenvolvimento de novos medicamentos, com ênfase para a produção de novos medicamentos e imunobiológicos, visando a promoção da saúde, prevenção e manutenção da vida dos 
portadores de doenças raras, além do estímulo e facilitação do registro e da entrada de medicamentos para o tratamento de doenças raras no mercado nacional e no SUS.

Por descumprimento de umas das condições apresentadas na Portaria $n^{\circ} .77$, "negociação para redução significante do preço", o medicamento eculizumabe sofreu ameaça de ser descontinuado do SUS pela Conitec, no qual uma consulta pública foi aberta para estudar a exclusão ou não do medicamento. Na consulta, portadores da doença, familiares e membros da classe médica opinaram sobre a exclusão do medicamento, apresentando justificativas e, em muitos casos contando, a experiência positiva com o medicamento. O impacto na saúde dos portadores é grande, uma vez que o medicamento proporciona independência de transfusão de sangue à grande parcela detentora da doença que afeta as células-tronco.

Em dado publicado pela Conitec, em 14 de setembro de 2021, foi apresentado que o Ministério da Saúde decidiu pela não exclusão da tecnologia, considerando que na perspectiva do paciente, a utilização do eculizumabe representou melhora expressiva dos sintomas da doença e da qualidade de vida, permitindo a retomada da vida social ativa, de trabalho regular, prática de esportes e um planejamento a longo prazo da vida. Além dos portadores, familiares participaram ativamente da consulta em prol da manutenção do fármaco, alertando que a utilização do ativo é capaz de salvar a vida e retomar a esperança.

Além dos serviços ofertados no serviço público de saúde, algumas associações, como a Associação Brasileira de Linfoma e Leucina (Abrale) e a AFAG, prestam grandes apoios aos portadores, na aquisição de medicamentos de alto custo via sistema judiciário, além do apoio durante o diagnóstico e o respectivo tratamento, com o objetivo de promover um acolhimento e inserção de pessoas com doenças raras e graves.

\section{Considerações finais}

A trajetória dos pacientes de doenças raras é longa e de alto impacto para vida dos pacientes e seus familiares.

Mesmo com diretrizes estabelecidas, muito pacientes portadores de doenças raras e seus respectivos familiares, portadores ou não de alguma comorbidade, recebem tratamento desigual no serviço de saúde. Não há equidade em relação aos direitos estabelecidos referente ao acesso a serviços de saúde, seja por falta de recursos para o tratamento da doença, ou a falta de conhecimento e preparo do profissional de saúde.

A incorporação da citometria de fluxo ao SUS foi de extrema importância, pois é um exame que exige um alto investimento, porém, é essencial para definir o diagnóstico, então dessa forma, o exame gratuito torna o acesso abrangente para pessoas com baixa renda ou em situações precárias.

Após a fase de diagnóstico, o paciente enfrenta uma grande dificuldade frente ao acesso a medicação. O alto custo do medicamento Eculizumabe torna o tratamento inacessível para os pacientes, sendo necessário o acesso por ação judicial, tornando o início do tratamento ainda mais tardio, devido ao longo processo entre a aprovação da compra do medicamento até realmente a entrega ao paciente. Há vários anos, o medicamento vendo movimentando um grande aparato judicial, realizando $100 \%$ das entregas do medicamento via judicialização em caráter público.

A incorporação do eculizumabe no sistema público de saúde, contemplando todo o diagnóstico e formas de tratamento da doença, traz aos pacientes esperança de um acesso eficiência e constante, com menos faltas do medicamento e sem a necessidade da judicialização. As contribuições dos pacientes a consulta pública realizada pela Conitec, refletem o medo e receio daqueles que necessitam diariamente do medicamento e lidam com a possibilidade de perderem a fonte da fluidez da saúde e melhora da qualidade de vida.

A medicação e, consequentemente, todo o amparo fornecido pelo SUS, juntamente com as equipes multidisciplinares que acompanham os pacientes, são direito de todos e devem sem mantidos. A esperança do portador, da família e daqueles que amam não pode morrer.

\section{Referências}

Anderson, M., Elliott, E. J., \& Zurynski, Y. A. 2013. Australian families living with rare disease: experiences of diagnosis, health services use and needs for psychosocial support. National Library of Medicine, 8(22), 1-9.

Arruda, M. M. A. S., Rodrigues, C. A., Yamamoto, M., \& Figueiredo, M. S. 2010. Hemoglobinúria Paroxística Noturna: da Fisiopatologia ao Tratamento. Revista da Associação Médica Brasileira, 56(2), 214-221.

Aureliano, W. A. 2018. Trajetórias Terapêuticas Familiares: doenças raras hereditárias como sofrimento de longa duração. Ciência \& Saúde Coletiva, 23(2), 369-379. 
Borowitz, M. J., Craig, F. E., Digiuseppe, J. A., Illingworth, A. J., Rosse, W., Sutherland, D. R., Wittwer, C. T., Richards, S. J., \& Society C. C. 2010. Guidelines for the diagnosis and monitoring of paroxysmal nocturnal hemoglobinuria and related disorders by flow cytometry. National Library of Medicine, 78(4), 211-230.

Brodsky, R. A. 2008. Advances in the diagnosis and therapy of paroxysmal nocturnal hemoglobinuria. National Library of Medicine, 22(2), 65-74.

Ehlert, L. R., Silva, C. L., \& Grando, A. C. 2021. A importância da citometria de fluxo no diagnóstico e monitoramento da hemoglobinúria paroxística noturna. Jornal Brasileiro de Patologia e Medicina Laboratorial, 57(1), 1-8.

Hillmen, P., Muus, P., Dührsen, U., Risitano, M. A., Schubert, J., Luzzatto, L., Schrezenmeier, H., Szer, J., Brodsky, R. A., Hill, A., Socié, G., Bessler, M., Rollins, S. A., Bell, L., Rother, R. P., \& Young, N. S. 2007. Effect of the complement inhibitor eculizumab on thromboembolism in patients with paroxysmal nocturnal hemoglobinuria. National Library of Medicine, 110(12), 4123-4128.

Hillmen, P., Muus, P., Röth, A., Elebute, M. O., Risitano, A. M., Schrezenmeier, H., Szer, J., Browne, P., Maciejewski, J. P., Schubert, J., Urbano-Ispizua, A., Castro, C., Socié, G., \& Brodsky, R. A. 2013. Long-term safety and efficacy of sustained eculizumab treatment in patients with paroxysmal nocturnal haemoglobinuria. National Library of Medicine, 162(1), 62-73.

Jang, J. H., Kim, J. S., Yoon, S., Lee, J., Kim, Y., Jo, D., Chung, J., Sohn, S. K., \& Lee, J. W. 2016. Predictive Factors of Mortality in Population of Patients with Paroxysmal Nocturnal Hemoglobinuria (PNH): Results from a Korean PNH Registry. National Library of Medicine, 31(2), 214-221.

Lei n. 10.938, de 19 de outubro de 2001. 2001. Dispõe sobre a Política Estadual de Medicamentos, e dá outras providências. Disponível em: https:/www.al.sp.gov.br/repositorio/legislacao/lei/2001/lei-1093819.10.2001.html.

Luz, G. S., Silva, M. R. S., \& DeMontigny, F. 2015. Doenças raras: itinerário diagnóstico e terapêutico das famílias de pessoas afetadas. Acta Paulista de Enfermagem, 28(5), 395-400.

Mathieu, D., Rahmouni, A., Villeneuve, P., Anglade, M. C., Rochant, H., \& Vasile, N. 1995. Impact of Magnetic Resonance Imaging on the Diagnosis of Abdominal Complications of Paroxysmal Nocturnal Hemoglobinuria. Blood, 85(11), 3283-3288.

Nishimura, J., Kanakura, Y., Ware, R. E., Shichishima, T., Nakakuma, H., Ninomiya, H., Decastro, C. M., Hall, S., ... \& Rosse, W. F. 200). Clinical course and flow cytometric analysis of paroxysmal nocturnal hemoglobinuria in the United States and Japan. National Library of Medicine, 83(3):193-207.

Pinto, M., Madureira, A., Barros, L. B. P., Nascimento, M., Costa, A. C. C., Oliveira, N. V., Albernaz, L., Campos, D. S., Horovitz, D. D. G., Martins, A. J., \& Moreira, M. C. N. 2019. Cuidado complexo, custo elevado e perda de renda: o que não é raro para as famílias de crianças e adolescentes com condições de saúde raras. Caderno de Saúde Pública, 35(9), e00180218.

Pirett, C. N. S., \& Medeiros, C. R. O. 2017. Doenças raras, medicamentos órfãos: reflexões sobre o dark side das organizações da indústria farmacêutica. Revista Brasileira de Estudos Organizacionais, 4(2), 437-460.

Brasil, Ministério da Saúde. 2014. Portaria n. 199, de 30 de janeiro de 2014. Institui a Política Nacional de Atenção Integral às Pessoas com Doenças Raras, aprova as Diretrizes para Atenção Integral às Pessoas com Doenças Raras no âmbito do Sistema Único de Saúde (SUS) e institui incentivos financeiros de custeio. Brasília: Ministério da Saúde.

Schrezenmeier, H., Muus, P., Socié, G., Szer, J., Urbano-Ispizua, A., Maciejewski, J. P., Brodsky, R. A., Bessler, M., Kanakura, Y., Rosse, W., Khursigara, G., Bedrosian, C., \& Hillmen, P. 2014. Baseline characteristics and disease burden in patients in the International Paroxysmal Nocturnal Hemoglobinuria Registry. Journal of the Ferrata-Storti Foundation, 99(5), 922-929.

SOLIRIS ${ }^{\circledR}$ (eculizumab) Bula do Profissional de Saúde. Bulário Eletrônico. Agência Nacional de Vigilância Sanitária (Anvisa); 2021. Recuperado de: http://www.anvisa.gov.br/datavisa/fi la_bula/index.asp.

\section{Minicurrículo}

Beatriz Boni de Mattos. Estudante do $8^{\circ}$ semestre (último) do curso de farmácia, na Universidade Anhembi Morumbi. 
Rodrigo Vinícius de Souza Carvalho. Estudante do $8^{\circ}$ semestre (último) do curso de farmácia, na Universidade Anhembi Morumbi. Atualmente atua como assistente de garantia de qualidade na Apsen Farmacêutica.

Anderson Scherer. Mestrando em Gestão de Alimentos e Bebidas, Universidade Anhembi Morumbi. Especialista em Gestão de Negócios e Marketing pela FETREMIS e Especialista em Planejamento de Vendas, pela Business School São Paulo. Bacharel em Hotelaria pela Universidade Anhembi Morumbi. Iniciei minha carreira na área da aviação com emissões internacionais. No decorrer de minha carreira profissional desenvolvi vários projetos de eventos culturais e sociais junto a Secretaria de Cultura do Estado de São Paulo. Atuante como Consultor Executivo de marketing e comunicação para empresas e indústria farmacêutica. Palestrante em diversas áreas de gestão e negócios, com ou sem foco em saúde, professor universitário ministrando aulas de Comunicação e Comportamento Clínico, Hospitalidade em Saúde, Gestão de Negócios e Pessoas, Projetos de Educação em Saúde, Políticas Pública de Saúde. Docente em pós-graduação ministrando disciplinas de Gestão em Saúde, Inovação em Saúde, Metodologia cientifica.

Como citar: Mattos, B.B., Carvalho, R.V.S., \& Scherer, A. 2021. A importância das políticas públicas para o tratamento da hemoglobinúria paroxística noturna. Pubsaúde, 8, a257. DOI: https://dx.doi.org/10.31533/pubsaude8.a257

Recebido: 28 set. 2021.

Revisado e aceito: 05 out. 2021.

Conflito de interesse: os autores declaram, em relação aos produtos e companhias descritos nesse artigo, não ter interesses associativos, comerciais, de propriedade ou financeiros que representem conflito de interesse.

Licenciamento: Este artigo é publicado na modalidade Acesso Aberto sob a licença Creative Commons Atribuição 4.0 (CC-BY 4.0). 